\title{
Más evidencia en contra del uso de vitaminas y antioxidentes en la prevención de enfermedades crónicas
}

MRC/BHF Heart Protection Study of antioxidant vitamin supplementation in 20.536 high-risk individuals: a randomised-placebo controlled trial. Heart Protection Study Collaborative Group. The Lancet 2002;360:23-33

\section{Objetivo}

Evaluar si el tratamiento suplementario con vitaminas antioxidantes reduce la tasa de incidencia de enfermedad vascular, cáncer y otros eventos adversos.

\section{Diseño}

Ensayo clínico aleatorizado controlado contra placebo dentro de un diseño factorial* con un seguimiento de cinco años. Se realizó una fase previa a la aleatorización con droga activa para aumentar la adherencia al estudio. La otra parte del estudio comparaba sinvastatina vs placebo1 (ver Artículo y comentario en estas páginas).

Lugar

69 hospitales dentro del Reino Unido

Pacientes:

Hombres (15.474) y mujeres (5.082) de 40 a 80 años de edad con factores de alto riesgo de padecer un evento vascular coronario en el transcurso de los siguientes cinco años: antecedentes de enfermedad coronaria u otra enfermedad arterial oclusiva, diabetes e hipertensión, un colesterol total en sangre mayor a $135 \mathrm{mg} / \mathrm{dL}$.

\section{Intervención}

Se aleatorizó a los pacientes a recibir tratamiento con vitaminas (600mg de vitamina $E, 250 \mathrm{mg}$ de vitamina $\mathrm{C}$ y $20 \mathrm{mg}$ de $\beta$-carotenos diarios) o placebo a través de un sistema central de aleatorización telefónica.

Medición de los resultados principales

El resultado principal se definió como el tiempo a la aparición de muerte por enfermedad vascular coronaria y no coronaria. Otros eventos evaluados fueron el tiempo a la aparición de cáncer u hospitalizaciones por trastornos psiquiátricos, causas respiratorias noneoplásicas, fracturas óseas y otras hospitalizaciones. El análisis se realizo por intención de tratar.

Resultados Principales

La tabla 1 muestra los principales resultados.

Conclusiones

En pacientes con alto riesgo cardiovascular, la suplementacion con vitaminas antioxidantes no mostró una reducción en la mortalidad global o en la incidencia de cualquier tipo de enfermedad vascular, cáncer u otro evento adverso mayor.

Tabla 1: principales resultados.

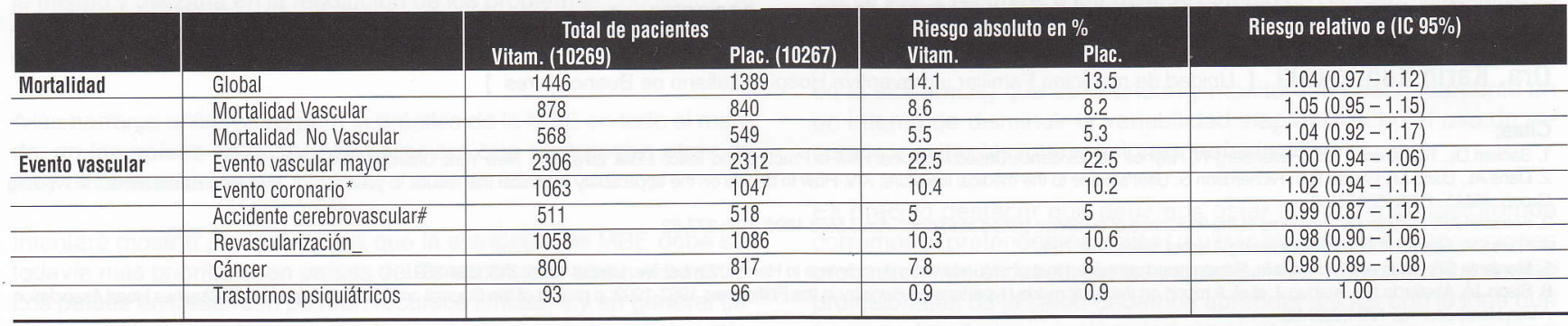

Fuente de financiamiento: *Infarto agudo de miocardio no fatal o muerte coronaria; \#Accidente cerebrovascular fatal y no fatal; _Revascularización coronaria y no coronaria

\section{Comentario}

El advenimiento de la hipótesis de modificación oxidativa y su relación con el mecanismo de aterosclerosis y envejecimiento tisular ha puesto en el tapete a las vitaminas antioxidantes como una de las potenciales intervenciones terapéuticas para contrarrestar esta reacción química. La década del noventa ha sido particularmente prolífica en la generación de estudios con el objetivo de testear esta hipótesis en el ámbito de la investigación clínica. Dentro de un amplio espectro de intervenciones terapéuticas que también incluyeron a la vitamina C; la vitamina E se ha focalizado en la prevención secundaria de las enfermedades vasculares (coronarias) y enfermedad de Alzheimer; y la vitamina A en la prevención primaria del cáncer. Estudios observacionales y de intervención se han extendido hasta nuestros días aportando datos a favor y en contra de la utilización de estos nutrientes naturales en la prevención de enfermedades crónicas $^{28}$. El presente estudio aporta un buen grado de evidencia (grado I*) en la recomendación de no suplementar con vitaminas la dieta habitual de un individuo con el propósito de prevenir eventos vasculares y no vasculares mayores y otras morbilidades. Los au- tores utilizaron gran parte de las estrategias disponibles para lograr una sólida validez interna a un menor costo (diseño factorial, población de alto riesgo, aleatorización estratificada*, fase de pre-aleatorización*, monitoreo anual de la tasa de eventos esperada, comité de evaluación independiente, etc.).

Quizá para aumentar la validez externa habría sido bueno conocer al menos los datos de los 11.609 pacientes que fueron pre-aleatorizados pero que por distintos motivos no fueron finalmente enrolados, de manera de poder evaluar la presencia y eventuales diferencias entre los participantes y no participantes (efecto placebo y efecto del voluntario sano*). La plausibilidad biológica de una hipótesis subyace en la relación de causalidad entre la potencial causa y su efecto. No conociendo como actúa el mecanismo de oxidación in vivo y con la cantidad de información hoy disponible, ¿hasta qué punto se debe seguir acumulando evidencia bajo esta hipótesis? ${ }^{9}$

Conclusión del comentador: Existe considerable evidencia para no suplementar con vitaminas la dieta habitual de un individuo con el propósito de prevenir eventos vasculares y otras morbilidades.

1. Heart Protection Study Collaborative Group. MRC/BHF Heart Protection Study of cholesterol lowering with simvastatin in 20536 high-risk individuals: randomised placebo-controlled trial. Lancet 360 (2002), pp. 7-22.

2.Jha, M. Flather, E. Lonn, M. Farkouh and S. Yusuf, The antioxidant vitamins and cardiovascular disease: a critical review of epidemiologic and clinical trial data. Ann Intern Med 123 (1995), pp. $860-872$.

3.L.H. Kushi, A.R. Folsom, R.J. Prineas, P.J. Mink, Y. Wu and R.M. Bostick, Dietary antioxidant vitamins and death from coronary heart disease in postmenopausal women. N Engl J Med 334 (1996), pp. $1156-1162 \mathrm{~K}$

4.Peto, R. Doll, J.D. Buckley and M.B. Sporn, Can dietary beta-carotene materially reduce human cancer rates?. Nature 290 (1981), pp. 201-208

5. St Do 5.N.G. Steph

781-786. 6.The Alpha-Tocopherol, Betac

330 (1994), pp. 1029-1035.
7.The Heart Outcomes Prevention Evaluation Study Investigators, Vitamin E supplementation and cardiovascular events in high-risk patients. N Engl J Med 342 (2000), pp. 154-160.

8.Collaborative Group of the Primary Prevention Project (PPP), Low-dose aspirin and vitamin E in people at cardiovascular risk: a randomised trial in general practice. Lancet 357 (2001), pp. 89-95. 9.D. Steinberg and J.L. Witztum, Is the oxidative modification hypothesis relevant to human atherosclerosis? Do the antioxidant trials conducted to date refute the hypothesis?. Circulation 105 (2002) pp. 2107-2111. 